\title{
Avaliação dos marcadores de lesão miocárdica solicitados em hospital paulista
}

\author{
Evaluation of markers of myocardial injury \\ requested in a hospital at São Paulo, Brazil
}

\author{
${ }^{1}$ Laboratório de Análises \\ Sacomã, São Paulo, SP, Brasil. CEP: 04231-030; ²Universidade Nove de Julho \\ (UNINOVE)- Curso de Farmácia-Bioquímica. Av. Dr. Adolpho Pinto, 109 Barra \\ Funda, São Paulo, SP. CEP:01156-050 Email:luvismari@gmail.com
}

\begin{abstract}
The evaluation of biochemical markers of myocardial necrosis is essential to the early diagnosis of acute myocardial infarction and to ensure a better patient prognosis. However, each marker has a profile that should suit the characteristics of clinical presentation that is being evaluated. So, the objective of the present study was to determine the profile of serum cardiac markers requested in the clinical laboratory of Heliopolis Hospital, and discuss the implications of such choices. By verifying the laboratory results maps, it was made a survey of biochemical markers of myocardial injury required in a period of three months, from patients admitted to the emergency department with symptoms suggestive of ischemic cardiac event. Among 2242 exams from 1084 patients, 47\% were for total CK, 43\% for CK-MB and $10 \%$ to troponins, with no requests of myoglobin dosage during the study period. Of the total number of tests ordered and changed, most refers to male patients. This study suggests that at the time of patient admission to the emergency department, a combination of myoglobin and a more specific marker of myocardial necrosis (CK-MB) should be requested to the early exclusion of a myocardial infarction. It would be, in addiction to effective to the diagnosis, more financially viable. In this context, a special attention should be given to women treated with ischemic symptoms, considering the high mortality rates associated to them.
\end{abstract}

Key Words: Diagnosis; Biochemical markers; Infarction

\section{RESUMO}

A mensuração dos marcadores bioquímicos de necrose miocárdica é essencial para diagnosticar precocemente o Infarto Agudo do Miocárdio e garantir um melhor prognóstico ao paciente. No entanto, cada marcador possui um perfil, que deve se adequar às características do quadro clínico que está sendo avaliado. Sendo assim, o objetivo do presente trabalho foi verificar o perfil de solicitações de marcadores cardíacos séricos junto ao laboratório clínico do Hospital Heliópolis, e discutir as implicações de tais escolhas. Por meio da verificação dos mapas de resultados do laboratório, foi feito um levantamento dos marcadores bioquímicos de lesão miocárdica solicitados, em um período de três meses, de pacientes admitidos no pronto socorro com sintomas sugestivos de um evento cardíaco isquêmico. Do total de 2242 exames provenientes de 1084 pacientes, 47\% eram para CK total, 43\% para CK-MB e 10\% para troponinas, não havendo solicitações de dosagem de mioglobina durante o período estudado. Do total de exames solicitados e alterados, a maioria referiu-se a pacientes do gênero masculino. O presente artigo sugere que no momento da admissão do paciente no serviço de emergência, haja a solicitação combinada da mioglobina e um marcador mais específico de necrose miocárdica (CK-MB) para a exclusão precoce de um infarto do miocárdio. Isto seria, além de eficaz para o diagnóstico, uma maneira mais viável financeiramente. Neste contexto, uma atenção especial deveria ser dada às mulheres atendidas com sintomas isquêmicos, tendo em vista a alta taxa de mortalidade a elas associada.

Palavras Chave: diagnóstico; marcadores bioquímicos; infarto 


\section{INTRODUÇÃO}

As Doenças Cardiovasculares (DC) são a principal causa de mortalidade no mundo, sendo as Doenças Isquêmicas do Coração, em particular o Infarto Agudo do Miocárdio (IAM), a causa mais comum (1). No Brasil, as Doenças do Aparelho Circulatório (CID-BR-10) foram responsáveis por $28,21 \%$ dos óbitos em 2012 (primeira causa de mortalidade proporcional). Destes, 84.121 casos foram decorrentes de IAM $(7,12 \%$ do total), sendo esta a segunda causa de morte mais frequente, de acordo com dados do Sistema Único de Saúde (Departamento de Informática do Sistema Único de Saúde [Datasus]) (2).

Nota-se, no entanto, uma tendência mundial de redução nessas taxas de mortalidade $(3,4)$. No Brasil, Mansur e Favarato (2012) publicaram um estudo onde se constatou a redução da mortalidade por doenças isquêmicas do coração, em homens e mulheres de todas as faixas etárias, no período compreendido entre 1990 a 2009, tanto no Brasil, como na região Metropolitana de São Paulo (5). No mesmo sentido, Baena et al (2012) observaram declínio da taxa de óbitos por IAM no período de 1998 a 2009 na cidade de Curitiba (6).

Um dos fatores que influenciam o melhor prognóstico em casos de IAM é o seu diagnóstico adequado e precoce. Seu diagnóstico inicial deve ser feito por uma combinação de sintomas, dados da anamnese e alterações eletrocardiográficas (ECG) características. No entanto, a confirmação do IAM depende da detecção de marcadores bioquímicos de lesão miocárdica, que são liberados na corrente sanguínea diante da lesão produzida pelo quadro isquêmico $(7,8)$. A avaliação destes marcadores é útil para estimar a extensão do infarto e auxiliar no prognóstico, no diagnóstico de reperfusão coronária e de infarto recorrente. Os marcadores bioquímicos mais utilizados são a creatinoquinase total (CK total) e sua fração MB (CK-MB), as troponinas cardíacas, e a mioglobina (7-9).

A CK-total é um indicador de lesão muscular não-específico para miocárdio. Sua fração CK-MB é o marcador tradicionalmente utilizado, embora tenha diversas limitações conhecidas (falsos positivos). Em casos de necrose miocárdica é possível observar elevação em seus níveis séricos entre 4-6 horas após o início dos sintomas. No diagnóstico de IAM, a mensuração de CK-MB no plasma por imunoensaio (CK-MB massa), em vez da sua atividade, apresenta maior sensibilidade e especificidade $(7,8)$.

As troponinas são consideradas marcadores precisos de IAM, porém sua elevação se dá entre 4 e 8 horas do início dos sintomas. Possuem como vantagem o fato de permanecerem em níveis elevados por tempo mais prolongado (até $24 \mathrm{~h}$ após o início dos sintomas). (7, 8).
A mioglobina, por sua vez, é uma das primeiras a apresentar elevação (1 a 2 horas após o início da lesão). Apesar de não ser um marcador específico para músculo cardíaco, valores normais de mioglobina podem auxiliar a afastar o diagnóstico de infarto. Valores alterados, por sua vez, necessitam de confirmação com outros marcadores para o diagnóstico adequado de IAM (7)

Muito se discute a respeito da utilização de kits para dosagem de troponina cardíaca de alta sensibilidade (hsTn), que são capazes de detectar até lesão miocárdica mínima, num momento precoce do atendimento $(7,10-$ 15). Se isto por um lado é positivo, devido à possibilidade de um diagnóstico rápido, por outro, existe um risco. Um estudo recente realizado em um hospital da Nova Zelândia mostrou que o número de testes positivos mais do que dobrou com o uso do ensaio de troponina cardíaca de alta sensibilidade. No entanto, $90 \%$ destes eram devidos a causas diferentes do infarto do miocárdio (10)

Assim, considerando que condições crônicas e comorbidades podem causar elevação, embora estável, dos níveis de hsTn, uma parceria entre o clínico e o laboratório é importante a fim de que haja o uso apropriado dos testes e uma interpretação precisa dos resultados. Também se faz necessário que sejam considerados outros aspectos, tais como o padrão de elevação dos marcadores e o cenário clínico, a fim de não sucumbir às armadilhas do excesso de diagnóstico (10-16).

A IV Diretriz da Sociedade Brasileira de Cardiologia sugeriu a utilização das troponinas T e I e da CK-MB massa como marcadores de lesão miocárdica. A dosagem de mioglobina deve ser feita para afastar o diagnóstico em pacientes com ECG que sugira IAM nas 6 primeiras horas após o início dos sintomas (8). Quanto mais precoce for feito o diagnóstico correto, mais rapidamente o tratamento terá início, e melhor a possibilidade de um bom prognóstico $(1,7,17,18)$. Neste sentido, a utilização adequada dos marcadores de lesão miocárdica é um ponto chave no sucesso do tratamento.

Sendo assim, o objetivo do presente trabalho é verificar o perfil de solicitações de marcadores cardíacos séricos junto ao laboratório clínico do Hospital Heliópolis, e discutir as implicações de tais escolhas.

\section{MÉTODO}

O presente estudo foi realizado no laboratório de análises clínicas do Complexo Hospitalar Heliópolis. Fundado em 1969, trata-se de um hospital geral público, mais popularmente conhecido como Hospital Heliópolis, mantido pelo governo do Estado de São Paulo. Com 330 leitos, seu atendimento abrange a população da zona sul de São Paulo e dos municípios de Santo André, São 
Bernardo do Campo, São Caetano do Sul, Diadema e Mauá, além de pacientes vindos de diversas regiões do País. Atende diversas especialidades (Cirurgia Geral, Hematologia, Reumatologia, Cirurgia de cabeça e pescoço, Cirurgia Torácica, Pneumologia, Ginecologia, Coloproctologia, Infectologia, Angiologia e Cirurgia Vascular, Cirurgia Plástica, Buco-Maxilo-Facial, Neurocirurgia, Cirurgia gastroenterológica, Clínica médica e Oncologia). Seu setor de emergência realiza em média 12 mil atendimentos mensais, sendo em sua maioria população de baixa renda.

É padrão solicitar aos pacientes que chegam ao setor de emergência com sintomas sugestivos de IAM a avaliação dos marcadores cardíacos de lesão miocárdica. Para isso, é coletada amostra de sangue de cada paciente (aproximadamente $10 \mathrm{~mL}$ em tubo seco com gel separador). As amostras são centrifugadas a 3000 R.P.M durante 8 minutos para separação do soro. A avaliação de CK total e CK-MB (atividade) é realizada pelo método enzimático colorimétrico (Aparelho CT600I e kit para dosagem, ambos da Empresa Wiener Lab). A troponina é avaliada pelo método imuno-enzimático com uma detecção final em fluorescência (ELFA). Os kits são da empresa SG Tecnologia Clínica (Kit Biomerieux).

A coleta de dados teve início após autorização pelo responsável do setor e aprovação pelo Comitê de Ética da Universidade Nove de Julho sob número de protocolo 11320/2012 (CAAE: 01904512.4.0000.5511).

Foram consultados os mapas do laboratório referentes aos marcadores bioquímicos de lesão miocárdica de pacientes adultos de ambos os gêneros admitidos no pronto-socorro no período de outubro a dezembro de 2011. Não foram avaliados outros resultados de exames dos referidos pacientes nem quaisquer outras informações, como uso de medicamentos ou presença de comorbidades ou menopausa. Os dados obtidos foram separados por gênero e tipo de marcador cardíaco solicitado, bem como a presença ou ausência de resultados alterados, de acordo com o padrão de referência de cada exame. Os resultados foram submetidos a análise estatística descritiva.

\section{RESULTADOS E DISCUSSÃO}

Houve a solicitação de 2242 dosagens de algum marcador bioquímico de lesão miocárdica, de material proveniente de 1084 pacientes. Destes, 593 (55\%) dos pacientes eram do gênero masculino e 491 (45\%) do gênero feminino. A Figura 1 ilustra a proporção de exames, de acordo com o marcador solicitado.

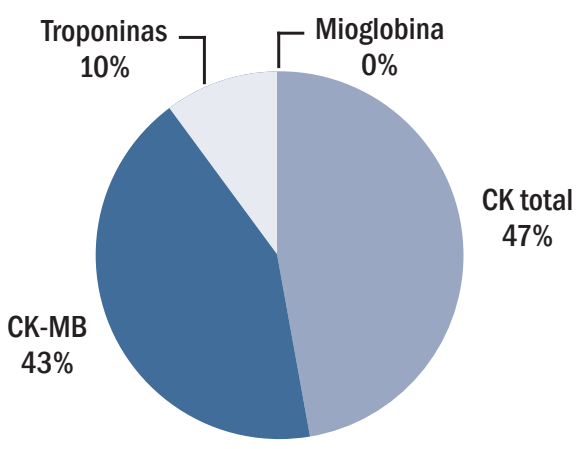

Figura1. Marcadores bioquímicos de lesão miocárdica solicitados ao laboratório clínico do Hospital Heliópolis de pacientes com sintomas cardíacos isquêmicos (outubro a dezembro de 2011)

Do total de 2242 exames, $47 \%$ (1052) eram para CK total, 43\% (966) para CK-MB e 10\% (224) para troponinas. Não houve solicitação de dosagem de mioglobina durante o período estudado.

A Figura 2 mostra a distribuição de resultados para cada marcador bioquímico de lesão miocárdica avaliado, de acordo com o gênero. Pode ser observada a presença de resultados alterados em 28,1\% (295) das dosagens de CK total, 39,4\% (371) das de CK-MB e em 24,43\% (164) das troponinas avaliadas. Além disso, é possível observar que, para todos os marcadores, o percentual de valores alterados (positivos) foi proporcionalmente maior em exames provenientes de pacientes do gênero masculino. Em resumo, a porcentagem de resultados alterados foi: para CK total de $35,4 \%$ (masculino) e 19,6\% (feminino); para CK-MB de 41,74 \% (masculino) e $36,53 \%$ (feminino); para troponinas de $30,97 \%$ (masculino) e 17,3\% (feminino).

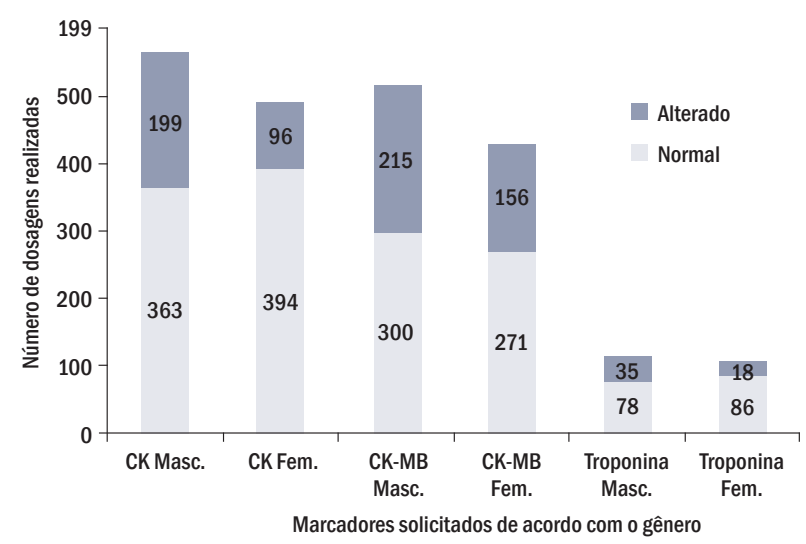

Figura 2. Distribuição de resultados para cada marcador bioquímico de lesão miocárdica avaliado, de acordo com o gênero

O principal marcador solicitado foi o CK total (47\%), que possui grande especificidade para lesões 
musculares, mas não é específico para o diagnóstico de lesão miocárdica. Tendo em vista o desenvolvimento de marcadores mais sensíveis e específicos, sua utilização deve ser considerada uma medida de exceção, reservada para o caso de indisponibilidade de marcadores mais modernos $(7,8)$. Os marcadores CK-MB e troponina corresponderam a $43 \%$ dos casos, indo ao encontro das principais recomendações das sociedades médicas $(7,8)$. Porém, as principais diretrizes da Sociedade Brasileira de Cardiologia recomendam a dosagem de mioglobina em adição a um marcador mais tardio (CK-MB) para pacientes que chegam precocemente à emergência (até 6 horas do início dos sintomas) $(7,8)$. Considerando que a procura por atendimento médico ocorre em média em um período de até quatro horas após o início dos sintomas (17,19-22), esta teria sido uma escolha adequada. No entanto, não houve um pedido sequer deste marcador, que apesar de não ser específico para lesão miocárdica, seria útil para descartar precocemente a ocorrência de necrose muscular. Vale a pena ressaltar que este não é um motivo econômico, tendo em vista a cobertura de todos esses marcadores tanto pelo SUS como pelos convênios.

No estudo de Cavalcanti et al (1998) com pacientes com sintomas isquêmicos a menos de $6 \mathrm{~h}$, a mioglobina mostrou ser mais sensível do que a CK-MB e com especificidade semelhante. Segundo os autores, a falta de conhecimento a respeito da mioglobina e a existência de tabus como a sua suposta baixa especificidade têm contribuído para o pouco emprego deste marcador (23).

Além disso, conforme a tabela SUS do período avaliado, o custo de cada dosagem era: $\mathrm{CK}$ total: $\mathrm{R} \$ 3,16$; CK-MB: R\$4,94; troponinas: $\mathrm{R} \$ 17,00$ e mioglobina: $\mathrm{R} \$ 11,42$. Dessa forma, solicitar mioglobina/CK-MB no primeiro exame, reservando a troponina para o segundo exame, além de eficaz para o diagnóstico, poderia ser uma maneira mais viável financeiramente.

Sabe-se que a maioria das mortes por IAM ocorre nas primeiras horas de manifestação da doença, sendo 40\%-65\% dos casos já na primeira hora (4). Além disso, quanto maior a demora no diagnóstico a partir do início dos sintomas, pior o prognóstico do paciente. Daí a importância de escolher os marcadores adequados para o diagnóstico de IAM, de acordo com o tempo de início dos sintomas. Também é fundamental conhecer de forma mais precisa o total de horas decorridas desde o início do quadro isquêmico. Neste sentido, tem sido proposto que a avaliação dos níveis plasmáticos de proteína $\mathrm{C}$ reativa (PCR) - um marcador de resposta inflamatória - fornece uma estimativa de total de tempo decorrido desde o início do IAM. De posse desta informação, é possível direcionar o tratamento do paciente de forma adequada (24). Além disso, existe uma correlação signi- ficativa entre níveis de PCR de alta sensibilidade (PCR-as) e de marcadores de lesão miocárdica, como Troponina I e CK-MB, podendo este ser utilizado como um importante marcador prognóstico. No entanto, a utilização do PCR-as ainda possui entraves econômicos que dificultam seu emprego (25).

Em relação ao gênero, houve um percentual maior de solicitações de exames para homens, os quais também apresentaram mais resultados alterados. Neste momento, é necessária uma reflexão: mulheres apresentam a primeira manifestação de doenças coronarianas em média dez anos mais tarde, além de um maior retardo na procura por assistência médica e maior mortalidade cardíaca que os homens (26-30).

Em artigo de revisão, Cantus e Ruiz (2011) avaliaram o perfil da cardiopatia isquêmica na mulher (28). Foi verificado que, em vários estudos, além do menor comparecimento aos serviços de emergência frente ao surgimento dos sintomas, sofrem mais com a demora no atendimento correto, onde lhe são aplicados menos testes diagnósticos, sendo seus sintomas muitas vezes confundidos com transtornos ansioso-depressivos. De fato, dentre as características dos sintomas femininos de IAM estão ausência de dor no peito (típico em homens) ou vago desconforto, dor nas costas, sensação de peso nos braços, vertigens, desconforto epigástrico, náuseas e vômitos (29).

As diferenças entre homens e mulheres em relação às doenças cardiovasculares são bem retratadas em artigo de revisão, no qual o autor ressaltou aspectos importantes a serem considerados (30). Além de um quadro sintomatológico mais silencioso, mulheres apresentam menos alterações eletrocardiográficas frente a um IAM, além de menor elevação dos marcadores bioquímicos tradicionais $\mathrm{CK}-\mathrm{MB}$ e troponinas. $\mathrm{O}$ autor sugeriu a utilização de PCR e do Peptídeo Natriurético tipo B (BNP) como ideal para o diagnóstico de IAM em mulheres. Esta informação, por sua vez, é extremamente relevante quando se verifica os dados do presente estudo, no qual houve um número maior de resultados alterados em pacientes do gênero masculino. Outros estudos seriam necessários a fim de verificar possíveis falsos negativos para IAM entre as mulheres.

Durante muito tempo acreditou-se em um possível efeito cardioprotetor dos estrogênios, que justificaria a maior ocorrência de IAM em mulheres após a menopausa. Em artigo de revisão publicado em 2014, o autor sugeriu que mulheres mais jovens e ou recentemente menopausadas poderiam obter proteção cardiovascular com a reposição hormonal. No entanto, seus efeitos benéficos em longo-prazo ainda são controversos (31). Portanto, ainda não há evidências suficientes que justifiquem a terapia de reposição hormonal na prevenção de doenças cardiovasculares em mulheres menopausadas (30). 
Diante destas diferenças de gênero frente a um IAM, os achados do presente trabalho somados à literatura ressaltam a necessidade de uma maior atenção aos eventos isquêmicos em mulheres.

\section{CONCLUSÃO}

Os dados obtidos permitem sugerir que na admissão do paciente no serviço de emergência, haja a solicitação de mioglobina e CK-MB, para a exclusão precoce de um infarto do miocárdio. E na reavaliação, que se dá em média 4 horas depois da primeira, poderia ser pedida apenas a troponina. Isso traria maior sensibilidade diagnóstica, que é importante para um

\section{REFERÊNCIAS}

1. Ruff CT, Baunwald E. The evolving epidemiology of acute coronary syndromes. Nat.Rev.Cardiol. 2011; 8:140-147.

2. BRASIL.Ministério da Saúde. Datasus. Sistema de Informação sobre Mortalidade. 2012 [Acesso em 2014 jul 17]. Disponível em: tabnet.datasus.gov.br/cgi/tabcgi.exe?sim/ cnv/obt10uf.def.

3. HPB. Health Promotion Board. Acute Myocardial Infarction Registry Report 2007 - 2012. [Acesso em 2014 jul 22]. Disponível em https://www.nrdo.gov.sg/uploadedFiles/NRDO/SMIR_Report_2007-2012_webv1.1.pdf

4. Tunstal-Pedoe H, Kuulasmaa K, Mahonen M, Tolonen H, Ruokokoski E, Amouyel P. Contribution of trends in survival and coronary event rates to changes in coronary heart disease mortality: 10 year results from 37 WHO MONICA Project population. Lancet.1999; 353(9146):1547-57.

5. Mansur AP, Favarato E. Mortalidade por Doenças Cardiovasculares no Brasil e na Região Metropolitana de São Paulo: Atualização 2011. Arq Bras Cardiol 2012;99(2):755-761

6. Baena CP, Olandoski M, Luhm KR, Constantini CO, Guarita-Souza LC, Faria-Neto JR. Tendência de Mortalidade por Infarto Agudo do Miocárdio e Curitiva (PR) no período de 1998 a 2009. Arq. Bras, Cardiol. 2012; 98(3):211-217.

7. Nicolau JC, Timerman A, Marin-Neto JA, Piegas LS, Barbosa CJDG, Franci A. Sociedade Brasileira de Cardiologia. Diretrizes da Sociedade Brasileira de Cardiologia sobre Angina Instável e Infarto Agudo do Miocárdio sem Supradesnível do Segmento ST. Arq Bras Cardiol 2014; 102(3Supl.1):1-61

8. Piegas LS, Feitosa G, Mattos LA, Nicolau JC, Rossi Neto JM, Timerman A, et al. Sociedade Brasileira de Cardiologia. Diretriz da Sociedade Brasileira de Cardiologia sobre Tratamento do Infarto Agudo do Miocárdio com Supradesnível do Segmento ST. Arq Bras Cardiol. 2009;93(6 Supl 2):e179-e264. bom prognóstico, além de menor custo para o Hospital. Neste contexto, uma atenção especial deve ser dada às mulheres atendidas com sintomas isquêmicos. A elas deveria ser destinado não apenas um olhar diferenciado em relação aos sintomas, como também um melhor acompanhamento do quadro, mesmo diante de resultados negativos dos marcadores bioquímicos tradicionalmente solicitados.

\section{AGRADECIMENTOS}

As autoras são gratas ao Laboratório de Análises Clínicas do Hospital Heliópolis por autorizar a realização deste estudo.
9. Volschan A, Polanczyk C, Clare C, Silva-Júnior DG, Mesquita ET, Abreu FB et al . I Diretriz de Dor Torácica na Sala de Emergência. Arq. Bras. Cardiol. 2002; 79 (2), $1-22$

10. Jairam S, Jones P, Samaraie L, Chataline A, Davidson J, Stewart R. Clinical diagnostics and outcomes for Tropo-

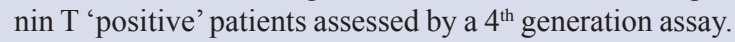
Emergency Medicine Australasia 2011; 23(4):490-501.

11. Uso da Troponina de Alta Sensibilidade para Diagnosticar Infarto Agudo do Miocárdio [editorial]. Rev Bras Cardiol. 2013;26(5):324

12. Cantelle CF, Lanaro R. Indicadores Bioquímicos do Infarto Agudo do Miocárdio. Rev.Ciênc.Saúde 2011; 1(3): 1-12.

13. Cervellin G, Lippi G. The clinical role of high-sensitive troponin assays: update, interpretation, and perspectives. Recenti Prog Med. 2010;101(11):423-8.

14. Sheng SC, Lu XM, Kang Y, Du YP, Wang GX. High-sensitivity cardiac troponin $\mathrm{T}$ is a predictor of recurrent acute coronary syndrome in patients with acute myocardial infarction. Genet Mol Res. 2014;13(2):3819-25. .

15. Giannitsis E, Kurz K, Hallermayer K, Jarausch J, Jaffe AS, Katus HA. Analytical validation of a high-sensitivity cardiac troponin T assay. Clin Chem. 2010;56(2):254-61.

16. Casagranda I, Lauritano EC. Diagnostic and prognostic significance of high sensitive troponin in chest pain. Eur Rev Med Pharmacol Sci. 2011 Jun;15(6):695-700.

17. Herlitz J, Wireklintsundström B, Bång A, Berglund A, Svensson L, Blomstrand C. Early identification and delay to treatment in myocardial infarction and stroke: differences and similarities. Scand J Trauma Resusc Emerg Med. 2010; 18:48.

18. Silva SH, Moresco RN. Biomarcadores cardíacos na avaliação da síndrome coronariana aguda. Scientia Medica (Porto Alegre) 2011; 21(3): 132-142. 
19. Soares JS, Souza NRM, Nogueira Filho J, Cunha CC, Ribeiro GS, Peixoto RS, Soares CEC, Soares LC, Reis AF, Faria CAC. Tratamento de uma Coorte de Pacientes com Infarto Agudo do Miocárdio com Supradesnivelamento do Segmento ST. Arq. Bras. Cardiol. 2009; 92(6):464-471

20. Franco B, Rabelo ER, Goldemeyer S, Souza EN. Pacientes com infarto agudo do miocárdio e os fatores que interferem na procura por serviço de emergência: implicações para a educação em saúde. Rev Latino-am Enfermagem. 2008; 16(3):414-418.

21. Denktas AE, Anderson V, McCarthy J, Smalling RW. Total ischemic time: the correct focus of attention for optimal ST-Segment elevation myocardial infarction care. JACC Cardiovasc Interv. 2011; 4(6):599-604.

22. J. K. Ghali, R. S. Cooper, I. Kowatly, and Y. Liao Delay between onset of chest pain and arrival to the coronary care unit among minority and disadvantaged patients. J Natl Med Assoc. Mar 1993; 85(3): 180-184.

23. Cavalcanti AB, Heinisch RH, Albino EC, Zunino JN. Diagnóstico do Infarto Agudo do Miocárdio. Valor da Dosagem de Mioglobina Sérica Comparada com a Creatinofosfoquinase e sua Fração MB. Arq Bras Cardiol. 1998; 70(2):75-80.

24. İlker Gül, Ahmet Çağrı Aykan, Tayyar Gökdeniz, Faruk Boyac1, Ezgi Kalaycığlu, Engin Hatem, Murat Bilgin, Şükrü Çelik. The relationship between C-reactive protein and the lapse of time since the onset of the symptoms after acute myocardial infarction: an prospective-observational study. Anadolu Kardiyol Derg 2014; 14(0): 000-000. [Acesso 2014 jul 21]. Disponível em http://www.anakarder.com/eng/makale/2904/106/Full-Text
25. Aseri ZA, Habib SS, Alhomida AS, Khan HA. Relationship of high sensitivity C-reactive protein with cardiac biomarkers in patients presenting with acute coronary syndrome. J Coll Physicians Surg Pak. 2014 Jun;24(6):38791.

26. Peixoto RTS, Peixoto ECS, Sena MA, Tedeschi AL, Borges IP, Rachid MBF. Influência do sexo na evolução imediata e a médio prazo após a intervenção coronariana percutânea primária e análise dos fatores independentes de risco para óbito ou eventos. Arq. Bras. Cardiol. 2006; 86(3): 211-218.

27. Mussi FC, Ferreira SL, Menezes AA. Vivências de mulheres à dor no infarto do miocárdio. Rev. Esc. Enferm. USP. 2006; 40(2):170-178. dx.doi.org/10.1590/S008062342006000200004 .

28. Cantus DS, Ruiz MDCS. A cardiopatia isquêmica na mulher. Rev. Latino-am. Enfermagem. 2011; 19(6):14621469.

29. Samantha J. Zbierajewski-Eischeid S, Loeb SJ. Recognizing myocardial infarction in women. 2010. [Acesso em 22 jul 2014]. Disponível em https://www.nursingcenter. com/_PDF_.aspx?an=00152193-201003001-00001.

30. Aziz F. Coronary Artery Disease in Women: An Unsolved Dilemma. J Clin Med Res. 2014;6(2):86-90.

31. Harman SM. Menopausal hormone treatment cardiovascular disease: another look at an unresolved conundrum. Fertil Steril. 2014 Apr;101(4):887-97. 